\title{
A simplified method to isolate rice mitochondria
}

\author{
Yanghong Xu' ${ }^{1}$, Xiaoyi Li' ${ }^{1}$ Jishuai Huang ${ }^{1}$, Leilei Peng ${ }^{1}$, Dinghui Luo', Qiannan Zhang ${ }^{1}$, Zhiwu Dan', \\ Haijun Xiao ${ }^{2}$, Fang Yang ${ }^{1 *}$ (D) and Jun $\mathrm{Hu}^{1 *}$ (D)
}

\begin{abstract}
Background: Mitochondria play critical roles in plant growth, development and stress tolerance. Numerous researchers have carried out studies on the plant mitochondrial genome structure, mitochondrial metabolism and nuclearcytoplasmic interactions. However, classical plant mitochondria extraction methods are time-consuming and consist of a complicated ultracentrifugation procedure with expensive reagents. To develop a more rapid and convenient method for the isolation of plant mitochondria, in this study, we established a simplified method to isolate rice mitochondria efficiently for subsequent studies.

Results: To isolate rice mitochondria, the cell wall was first disrupted by enzymolysis to obtain the protoplast, which is similar to animal mitochondria. Rice mitochondria were then isolated with a modified method based on the animal mitochondria isolation protocol. The extracted mitochondria were next assessed according to DNA and protein levels to rule out contamination by the nucleus and chloroplasts. Furthermore, we examined the physiological status and characteristics of the isolated mitochondria, including the integrity of mitochondria, the mitochondrial membrane potential, and the activity of inner membrane complexes. Our results demonstrated that the extracted mitochondria remained intact for use in subsequent studies.
\end{abstract}

Conclusion: The combination of plant protoplast isolation and animal mitochondria extraction methods facilitates the extraction of plant mitochondria without ultracentrifugation. Consequently, this improved method is cheap and time-saving with good operability and can be broadly applied in studies on plant mitochondria.

Keywords: Mitochondria isolation, Protoplast, Rice

\section{Background}

Mitochondria were first discovered in 1850 and are considered to have evolved from the engulfment of an $\alpha$-proteobacterium by a precursor of the modern eukaryotic cell [1]. The differences between plant mitochondria and animal mitochondria are small regarding their size and function since both of them evolved from the

\footnotetext{
*Correspondence: fyang@whu.edu.cn; junhu@whu.edu.cn

1 State Key Laboratory of Hybrid Rice, Engineering Research Center for Plant Biotechnology and Germplasm Utilization of Ministry

of Education, College of Life Sciences, Wuhan University, Wuhan 430072, Hubei, China

Full list of author information is available at the end of the article
}

same microorganism [2, 3], but the plant mitochondrial genome can be 100 times larger than those of animals [4, 5].

Mitochondria produce more than $90 \%$ of the cellular energy (ATP) required for an organism's growth, reproduction, and maintenance [6]. In plants, dysfunction of the mitochondria always leads to retardation of plant growth, hypersensitivity to disease, embryo lethality, pollen abortion and other disorders [7-9]. The mitochondrion is also vital to the mammal, as deficiency in mitochondrial function results in many serious diseases, such as Alzheimer's, Parkinson's, and Huntington's disease [10-12]. Recently, a growing number of researchers have focused on studies of the mitochondria to explore 
an efficient therapeutic approach to deal with existing and emerging mitochondrial diseases [13, 14]. A large number of studies have revealed that the reason for mitochondrial deficiency might be the incompatibility between mitochondria and the nucleus or mutations in the mitochondrial genome. Generally, these dysfunctions result from disorders of transcriptional, post-transcriptional and translational regulation. Many groups have performed plant mitochondrial omics studies, and the interaction between the nucleus and the mitochondrion has contributed to speciation and cytoplasmic male sterility, which could be applied to explore crop hybrid vigor [15-22].

The classic method of rice mitochondria isolation was reported a dozen years ago and has been broadly applied in many labs, including ours [23]. Nevertheless, there are still some flaws in this method. First, it needed a large amount of plant material, since there is a great loss during the extraction process. Second, it also requires heavy labor to grind the materials. Third, the preparation of the Percoll gradient solution, an expensive reagent, is also laborious and time-consuming. More importantly, a high-speed centrifuge and an ultra-speed centrifuge are indispensable. All these requirements prohibit most labs from isolating plant mitochondria and conducting mitochondria-associated studies.

In this study, we established an improved method to extract rice mitochondria. In this procedure, we excluded the heavy labor, the expensive equipment and reagents, and reduced the requirement for the enormous amount of material. To confirm the purity of the isolated plant mitochondria, we assessed the extracts at the DNA and protein levels. The results of the proteinase digestion assay and the electron scan microscopy observation also confirmed the intact structure of the mitochondria. The mitochondrial membrane potential and functional activity of the inner membrane electron transport chain (ETC) complex were checked to assure the suitability of the mitochondria for subsequent studies.

\section{Results}

\section{Workflow of the mitochondria isolation}

In this study, we improved the mitochondria extraction method by combining the traditional method of plant protoplast isolation and the mammalian mitochondria extraction protocol with slight modifications [24, 25] (Fig. 1).

First, the rice seeds were cultured in 1/2 MS medium at $25{ }^{\circ} \mathrm{C}$ in a dark environment for 10 days to avoid bacterial contamination. Next the 10-day-old seedlings were cut into $\sim 0.5 \mathrm{~mm}$ pieces, which were dipped in hyperosmotic buffer for $10 \mathrm{~min}$, followed by further incubation with enzyme buffer for $5 \mathrm{~h}$ at $28{ }^{\circ} \mathrm{C}$ or overnight at $25{ }^{\circ} \mathrm{C}$
[26]. The results suggested that the treatment overnight at $25{ }^{\circ} \mathrm{C}$ could yield more viable protoplasts (Table 1). Subsequently, the protoplasts were further collected by centrifugation at $600 \mathrm{~g}$ for $5 \mathrm{~min}$ and resuspended gently in mitochondrial isolation buffer (MIB). The suspension was incubated at $4{ }^{\circ} \mathrm{C}$ for $1 \mathrm{~h}$, and the protein inhibitor PMSF was added to a final concentration of $1 \mathrm{mM}$ to prevent degradation of the mitochondria by cytoplasmic proteinases. To obtain the highest yield of the mitochondria without destroying its integrity, the optimal amount of sample to homogenize was determined. Finally, the sample was centrifuged at $600 \mathrm{~g}$ for $10 \mathrm{~min}$, the supernatant was decanted into another tube, and the mitochondria were collected by centrifugation at $11,000 \mathrm{~g}$ for $10 \mathrm{~min}$. By this procedure, we obtained approximately $10 \mathrm{mg}$ of mitochondria from $12 \mathrm{~g}$ of seedlings.

\section{The purity of the mitochondria}

To assess the purity of our isolated mitochondria, we first employed a PCR assay to assess at the DNA level. COX III, rubisco and actin were selected as the mitochondrial, chloroplast and nuclear marker genes, respectively. No actin and rubisco PCR products were observed even after 35 cycles of amplification, indicating that DNA isolated from purified mitochondria were free from the nuclear and chloroplast DNA contamination (Fig. 2a).

Furthermore, several specific commercial antibodies, anti-histone, anti-rubisco, anti-catalase and anti-VDAC (voltage-dependent anion channel) were applied to estimate contamination from the nucleus, chloroplast, cytoplasm and mitochondrion, respectively. Three types of components were further analyzed: the supernatants that contained the cytoplasmic substance in step 10 (S10, outlined in the Sect. "Materials and methods") were collected, and the precipitates in step 9 (S9, outlined in the Sect. "Materials and methods") that contained the cell lysates and the intact protoplasts were retained. The results showed that all of the cell organelle maker proteins could be detected in the total cells, indicating that the protoplast was intact (Fig. 2b). In the mitochondria fraction, only the marker protein VDAC could be detected, indicating that the mitochondria were solidly enriched. No evidence of peroxisomes, rubisco or histones was detected in the mitochondria fraction, suggesting that most of organelles were removed (Fig. 2). All these results demonstrated that the mitochondria had been isolated with a high purity.

\section{Determination of the mitochondrial integrity}

To determine whether the extracted mitochondria were intact, we examined the integrity of the mitochondrial membrane via proteinase $\mathrm{K}$ treatment. The inner membrane proteins are more resistant against proteinase 
a

Cultivate the rice on the sterilized rooting medium under dark conditions at $25^{\circ} \mathrm{C}$ for 10 days

b

Dip the chopped seedlings in the balance buffer for $10 \mathrm{~min}$

c

d

e Examine the completely lysed
protoplasts by microscope

f

\begin{tabular}{l} 
Examine the incompletely lysed \\
protoplasts by microscope \\
\hline
\end{tabular}

Precipitate the mitochondria by differential centrifugation

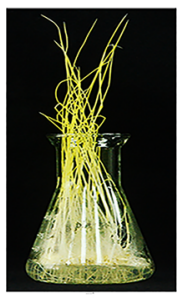

$$
\text { ) }
$$

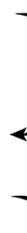

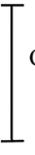

Chopped region

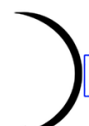

Cut the seedlings into pieces with razor

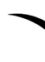

Incubate the chopped seedlings with enzyme buffer at $25^{\circ} \mathrm{C}$
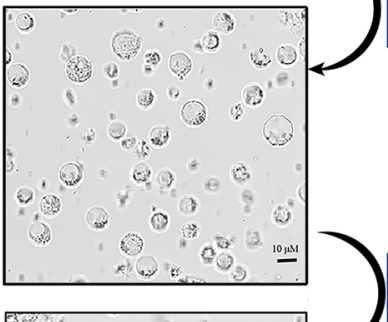

Incubate the rice protoplasts with MIB at $4^{\circ} \mathrm{C}$
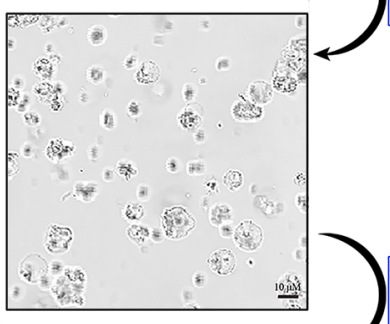

Homogenize the lysed protoplast suspension 20 times

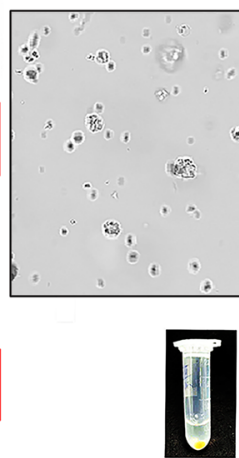

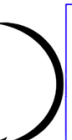

Centrifuge homogenated solution for $10 \mathrm{~min}$ at $600 \mathrm{~g}$, decant the supernatant into new tube and centrifuged for $10 \mathrm{~min}$ at $11,000 \mathrm{~g}$

Fig. 1 Procedure for mitochondria isolation from rice seedlings. The procedure contains two parts: the protoplasts extraction (a-c) and the mitochondria isolation from protoplasts $(\mathbf{d}-\mathbf{f})$. The reverse double arrows show the times consumed by each part. MIB: mitochondria isolation buffer. Bars, $10 \mu \mathrm{m}$

Table 1 Yields of protoplasts and mitochondria from seedlings treated with enzyme buffer under different incubation temperatures and times

\begin{tabular}{|c|c|c|c|c|c|c|}
\hline \multirow[t]{2}{*}{ Dataset } & \multicolumn{3}{|l|}{$25^{\circ} \mathrm{C}$} & \multicolumn{3}{|l|}{$28^{\circ} \mathrm{C}$} \\
\hline & $5 \mathrm{~h}$ & $8 \mathrm{~h}$ & $12 \mathrm{~h}$ & $5 \mathrm{~h}$ & $8 \mathrm{~h}$ & $12 \mathrm{~h}$ \\
\hline Protoplasts (Number) & $3.75 \times 10^{7}(0.75)$ & $7.55 \times 10^{7}(1.65)$ & $5.10 \times 10^{7}(0.40)$ & $5.40 \times 10^{7}(0.54)$ & $6.00 \times 10^{7}(1.20)$ & $1.65 \times 10^{7}(0.54)$ \\
\hline Mitochondria (mg) & $7.00(3.12)$ & $12.00(6.54)$ & $8.50(5.68)$ & $9.00(3.00)$ & $9.00(0.00)$ & $4.85(3.76)$ \\
\hline
\end{tabular}

The numerical values in each cell represent the mean and standard deviation (in parentheses) 


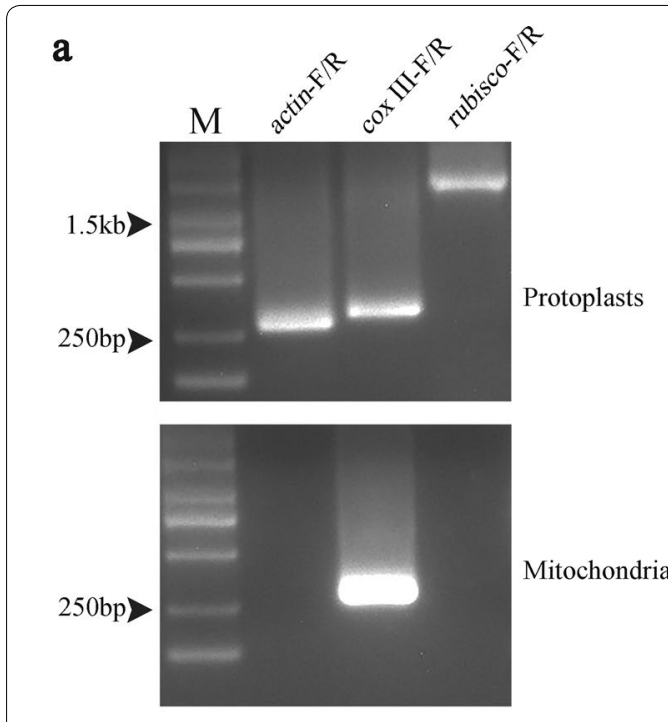

b

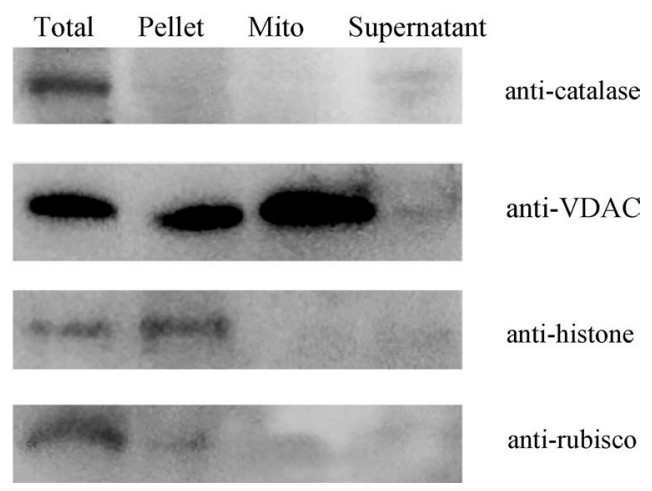

Fig. 2 Determination of mitochondrial purity. a The existence of the nuclear genome, chloroplast genome and the mitochondrial genome was examined by actin, rubisco and cox III, respectively. M, DNA 2000 marker. b Four components of cells were collected for this experiment. The total lane indicates the protoplasts, the pellet lane indicates lysates from S9 (as outlined in the Sect. "Materials and methods"), the mito lane indicates the mitochondria and the supernatant lane indicates the cytoplasmic, which was derived from the supernatant in $\mathrm{S} 10$ (as outlined in the Sect. "Materials and methods"). Rubisco, histone, VDAC and catalase were examined as the marker proteins of the chloroplast, nucleus, mitochondria and peroxisome, respectively

$K$ than those located on the outer membrane in the intact mitochondrion, while in the fractured mitochondrion, the two types of proteins show identical degradation patterns when exposed to proteinase $\mathrm{K}$. The result showed that the outer membrane protein VDAC was degraded under the proteinase treatment, while no degradation occurred for the inner membrane protein NADH dehydrogenase subunit 3 (NAD3) with the increasing concentration of proteinase K (Fig. 3a). In contrast, both VDAC and NAD3 exhibited identical

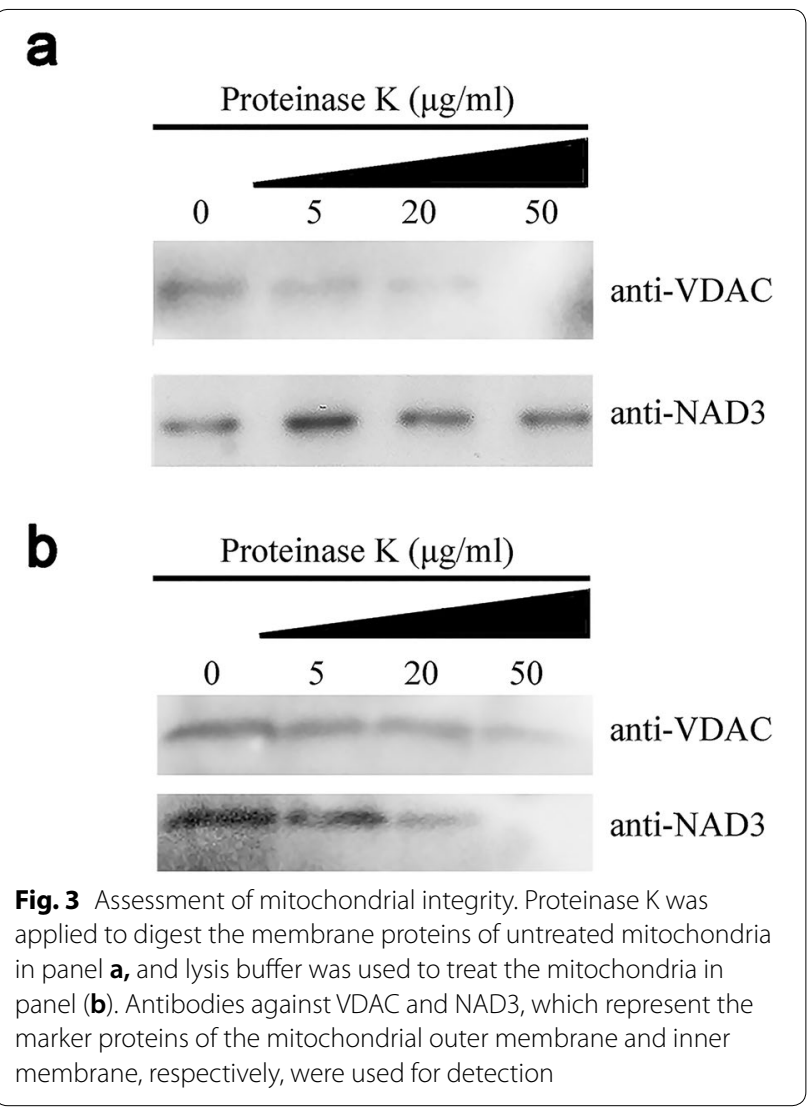

degradation patterns when the mitochondria were treated with proteinase $\mathrm{K}$ after resuspension in full lysis buffer (FLB), suggesting that the membranes were destroyed completely (Fig. $3 \mathrm{~b}$ ). We also used thermolysin to assess the integrity of the mitochondrial membrane. In the chloroplasts, thermolysin can digest the outer membrane peptide but it cannot penetrate into the intermembrane space [27]. Therefore, we proposed that thermolysin would have a similar biological effect on the mitochondrial outer membrane. Under treatment with an increasing thermolysin concentration, we observed the continuous degradation of VDAC, whereas the inner membrane marker protein COXII remained stable (Additional file 1: Fig. S1). This distinct degradation pattern implied that our extracted mitochondria were intact.

To further observe the morphology of the mitochondria, they were also examined by transmission electron microscopy (Fig. 4). We observed numerous mitochondria that contained a high electron density, indicating that the isolated mitochondria preserved a high viable activity [28]. All of these results suggested that the mitochondria were well enriched and that the structure remained intact, which allows for their use in further research. 


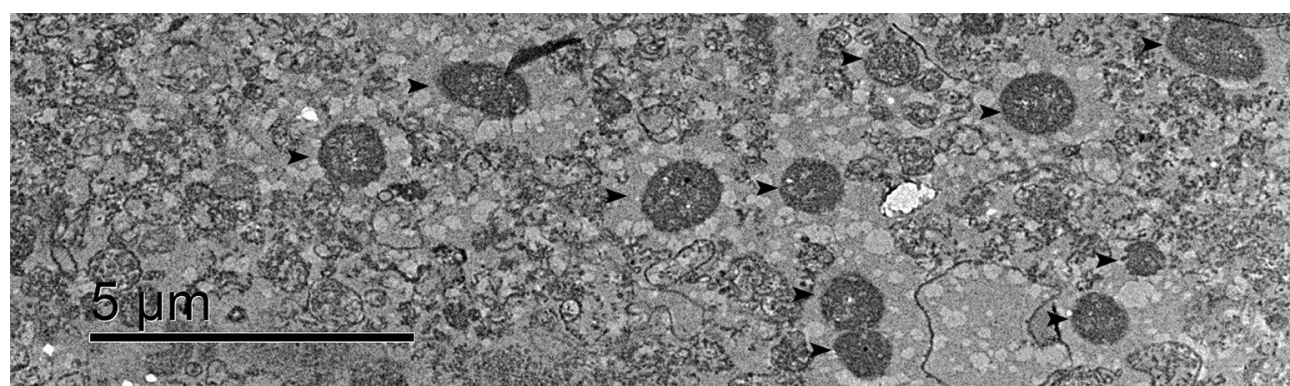

Fig. 4 Observation of mitochondria with transmission electron microscopy. Isolated mitochondria were observed to be enriched; the black arrows indicate the intact mitochondria with high electron-density in the matrix. Bars, $5 \mu \mathrm{m}$

\section{The mitochondrial membrane potential assay}

A previous study showed that fluorescence substrate JC-1 can specifically aggregate in the viable mitochondria and emit red fluorescence signals, while in apoptotic mitochondria, JC-1 exist in a monomeric state, which emits green fluorescence [29]. Therefore, JC-1 was then used to analyze the mitochondrial membrane potential. The rice protoplasts were incubated with $\mathrm{JC}-1$ according to the protocol of the mitochondrial membrane potential detection kit. We also treated the protoplasts with carbonyl cyanide 3-chlorophenylhydrazone (CCCP), which is a mitochondrial membrane potential inhibitor, before staining with JC-1. The results showed that the protoplasts treated with CCCP exhibited stronger green signals than the untreated protoplasts (Fig. 5a), suggesting that more apoptotic mitochondria were generated when

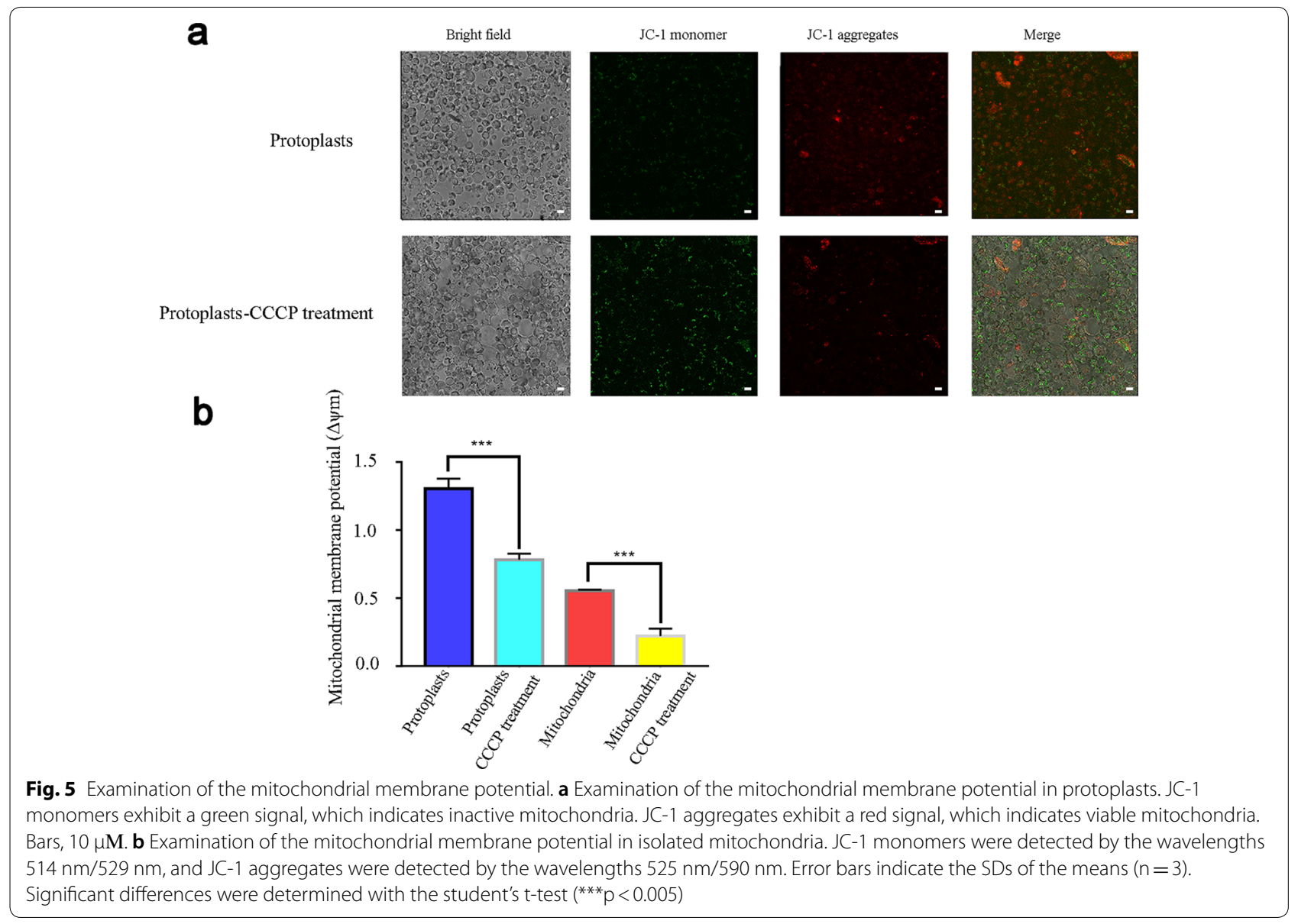


the protoplasts were treated with the mitochondrial membrane potential inhibitor.

Moreover, we used the Cytation 3 Multimode Reader to detect the fluorescence signal emitted by JC-1 aggregates or monomers, and the ratio of red and green fluorescence was calculated to indicate the mitochondrial membrane potential. As a control, the results demonstrated that CCCP-treated protoplasts showed a significantly decreased mitochondrial membrane potential compared to the untreated protoplasts. We then further assessed the membrane potential of isolated mitochondria, and the results showed that the membrane potential significantly declined, indicating that the isolated mitochondria kept its potential to some degree (Fig. 5b).

\section{The inner membrane complex activity of mitochondria}

The activities of the ETC complex are very essential for the organism. Therefore, an activity assay of the mitochondrial inner membrane complex also needed to be performed in this study, even though the integrity of the mitochondria was confirmed.

We investigated the activity of the isolated mitochondria using three different mitochondria inhibitors: rotenone $(0 \mu \mathrm{g} / \mathrm{ml}, 3 \mu \mathrm{g} / \mathrm{ml}, 6 \mu \mathrm{g} / \mathrm{ml}, 9 \mu \mathrm{g} / \mathrm{ml})$, which inhibits the ETC complex I, malonate $(0 \mathrm{mg} / \mathrm{ml}, 0.5 \mathrm{mg} /$ $\mathrm{ml}, 2.5 \mathrm{mg} / \mathrm{ml}, 5 \mathrm{mg} / \mathrm{ml}$ ), which inhibits the ETC complex II, and sodium azide $(0 \%, 0.1 \%, 1 \%, 2 \%)$, which acts on the ETC complex IV [30-32]. All three inhibitors were incubated with the extracted protoplasts for $30 \mathrm{~min}$ at $25{ }^{\circ} \mathrm{C}$ before isolating the mitochondria. The complex activity was assayed with the mitochondrial complex activity assay according to the manufacturer's instructions with slight modification. The results showed that the activities of the mitochondrial inner membrane ETC complexes significantly declined under the different inhibitor treatments (Fig. 6). In addition, the complex activities were severely decreased with the increased concentration of inhibitor, which suggested that the isolated mitochondria could be used in subsequent studies.

\section{Discussion}

\section{The simplified mitochondria isolation procedure}

Plant mitochondria are difficult to isolate due to the presence of the cell wall, which is absent in the animal cell. The basic idea of our method was to obtain the protoplasts, which were similar to the animal cells, and then the mitochondria could be extracted easily according to the slightly modified mammalian mitochondrial isolation methods. We chose 10-day-old rice seedlings from which to isolate the protoplasts, since the plant cell undergoes active mitosis at this young stage and the cell wall can be easily degraded by cellulase and macerozyme. The cell structure of the extracted protoplast is similar to that of

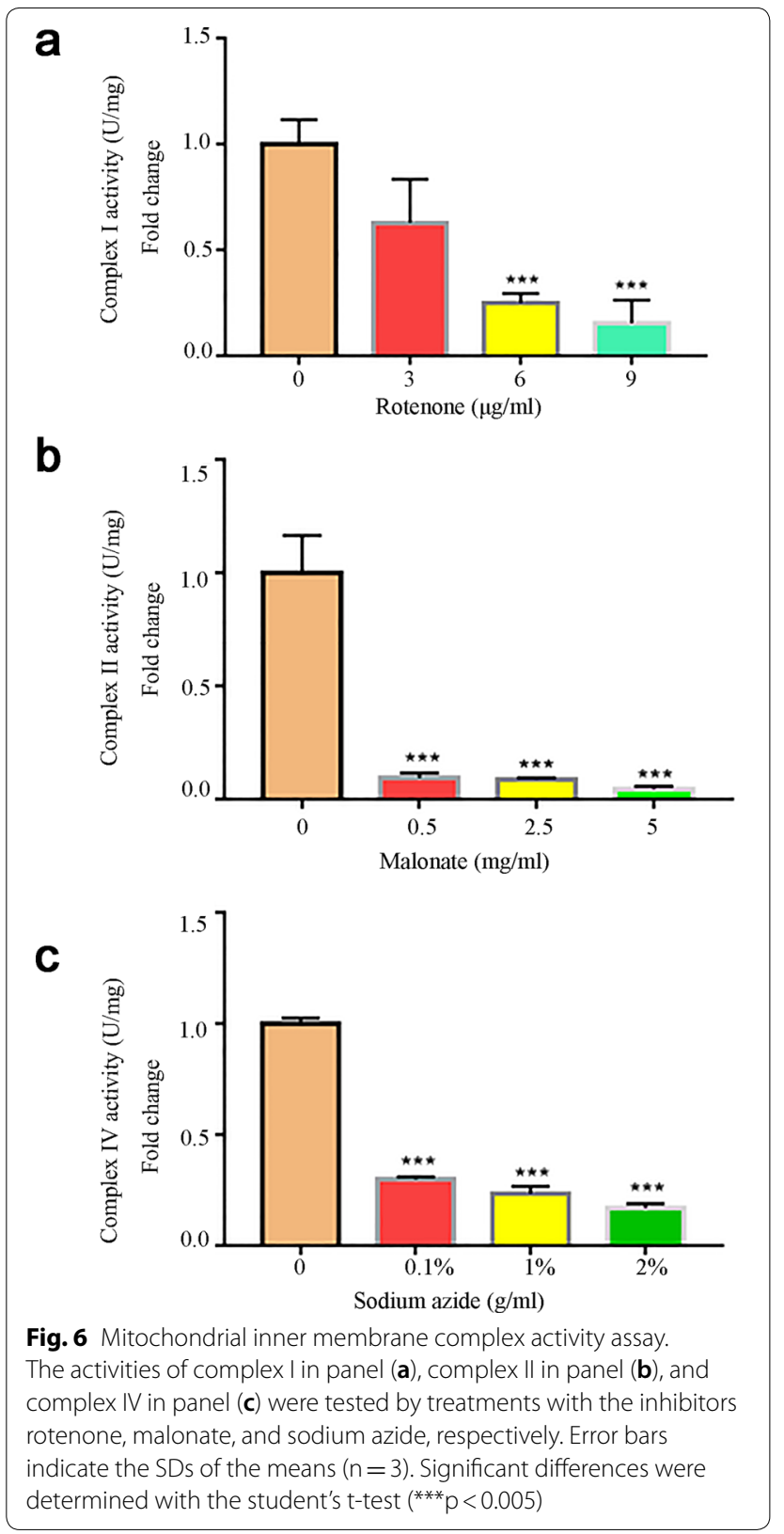

the animal cell, so it is reasonable to isolate the plant cell mitochondria by applying a homogenizer to burst the cell membrane in MIB. In this way, the mitochondria can be easily isolated from the seedlings. In addition, we also successfully obtained abundant rice protoplasts from the callus (Additional file 1: Fig. S2), suggesting that the callus can be used to isolate the rice mitochondria with this method.

Compared to the traditional rice mitochondria extraction methods that require strenuous labor to prepare the Percoll density gradient solution and multiple differential centrifugations, this improved method simplified 
the solution preparation by excluding the difficult preparation of a large amount of plant material and Percoll density gradient solution. Since most of the experimental time was spent in the enzyme solution incubation period, this method reduced the labor largely compared to the traditional mitochondria isolation method. The extraction of the mitochondria is not only important for the morphology and integrity of mitochondria but also essential for their activities and function. In this method, we greatly reduced the operation time to keep the mitochondria intact. In addition, the expenditure was also decreased since the expensive reagent Percoll and the ultra-speed centrifuge equipment are not necessary, which are not always affordable in many labs.

\section{Tips for a high yield of mitochondria}

Based on our results, we believe that the successful acquisition of plant protoplasts is the guarantee for extraction. To harvest more mitochondria, the high yield of protoplasts is the key step. Although the chopped rice seedlings were suggested to be incubated with enzyme buffer for $5 \mathrm{~h}$ at $28{ }^{\circ} \mathrm{C}$ or overnight at $25{ }^{\circ} \mathrm{C}$, we recommend treating the young seedlings for $8 \mathrm{~h}$ at $25{ }^{\circ} \mathrm{C}$ (Table 1 ). For the callus, the incubation time can be extended to avoid the incomplete cell wall degradation. Generally, the shorter the incubation time, the higher the incubated temperature that is needed for the enzyme reaction. To yield more protoplasts from different tissues or species, various temperatures or times should be tested. The protoplasts are then lysed in MIB, and the incubation time is changeable when combined with the homogenization time. The longer the MIB incubation time, the less time for homogenization. Otherwise, both over-incubation and over-homogenization can result in broken mitochondria and inefficient mitochondria yields (Additional file 1: Fig. S3).

\section{The extracted mitochondria remained active}

Since isolated mitochondria are vulnerable to damage and a fractured mitochondria structure greatly affects the results of experiments, in this study, we performed proteinase digestion experiments to examine the integrity of the mitochondrial structure. The mitochondrial marker proteins in the inner membrane and matrix were protected from digestion by proteinase $\mathrm{K}$ and thermolysin, which established that the isolated mitochondria and the outer membranes were intact. Furthermore, both of the CCCP-treated protoplasts and isolated mitochondria exhibited a significantly decreased mitochondrial membrane potential, which demonstrated that the mitochondria still kept their membrane potential in our extraction procedure.
We also examined the activity of ETC complexes in the inner membrane mitochondria. All of the tested mitochondrial ETC complexes in the inner membrane revealed a decline in activity, which exhibited a negative relationship with the increasing concentration of toxicant, indicating that the function of the mitochondrial ETC complexes was blocked by the toxicant. All of the results demonstrated that the isolated mitochondria could be used for biochemical analyses.

\section{Conclusions}

In summary, the simplified method proposed in this study provides researchers an alternative way to isolate rice mitochondria. As the protoplasts of different plants are accessible [33-35], the mitochondria extraction method can be applied to other plants without preparing abundant plant materials. Compared to the traditional mitochondrial extraction methods, this mitochondria isolation method does not include expensive reagents, instruments and complicated steps. Moreover, the improved mitochondria extraction method can greatly shorten the time and save labor. All these improvements demonstrated that this simplified method has wide potential applications for plant mitochondria extraction for subsequent research.

\section{Materials and methods \\ Plant materials}

Two hundred rice seeds were prepared by dehulling the seeds with a sheller (Zhejiang Cheering Sewing Machine Company, China), followed by sterilization with $75 \%$ ethanol for 1-2 min. The rice was rinsed with sterilized water, followed by treatment with $0.15 \% \mathrm{HgCl}_{2}$ for $15 \mathrm{~min}$. The rice was cultivated on sterilized rooting medium for sprouting or induction medium for callus generation under dark conditions at $25^{\circ} \mathrm{C}$ for 10 days and 20 days, respectively.

\section{Protocol for the mitochondria isolation}

The improved plant mitochondria isolation method was based on protoplast extraction and lysis, and mitochondria were further isolated through differential centrifugation with a protocol that consisted of the following 10 steps:

S1. Etiolated seedlings were collected, and the seeds were removed, after which the seedlings were dipped in balance buffer ( $0.6 \mathrm{M}$ mannitol). For the callus, the newly generated calluses were chosen and dipped in balance buffer.

S2. The middle region was selected, and the seedlings were chopped into $0.5 \mathrm{~mm}$ pieces with a razor 
blade, which were then put in balance buffer for at least $10 \mathrm{~min}$.

S3. Chopped seedlings or calluses were filtered with a nylon net (300 mesh, $0.05 \mathrm{~mm}$ aperture) and transferred into enzyme buffer (10 mM MES, pH 5.7, 0.6 M mannitol, $0.1 \%$ BSA, $1.5 \%(\mathrm{w} / \mathrm{v})$ cellulase R10 (YakultHon sha, Tokyo, Japan), 0.75\% (w/v) macerozyme R10 (Yakult Honsha), $1 \mathrm{mM} \mathrm{CaCl} 2,1 \mathrm{mM}$ $\beta$-mercaptoethanol, $50 \mu \mathrm{g} / \mathrm{ml}$ carbenicillin) in a water bath for $10 \mathrm{~min}$ at $55{ }^{\circ} \mathrm{C}$, after which the mixture was allowed to cool to room temperature.

S4. The mixture was shaken at $80 \mathrm{rpm}$ for 5 hours at $28{ }^{\circ} \mathrm{C}$ in a dark environment.

S5. Chopped seedlings or calluses were filtered, and the filtrates were collected in a $7 \mathrm{ml} \mathrm{EP}$ tube and centrifuged at $600 \mathrm{~g}$ for $3 \mathrm{~min}$ at $25^{\circ} \mathrm{C}$.

S6. The pellet was resuspended with W5 solution (2 mM MES, pH 5.8, $154 \mathrm{mM} \mathrm{NaCl}, 125 \mathrm{mM} \mathrm{CaCl}_{2}$, $5 \mathrm{mM} \mathrm{KCl}, 5 \mathrm{mM}$ glucose) and centrifuged at $600 \mathrm{~g}$ for $3 \mathrm{~min}$ at $25{ }^{\circ} \mathrm{C}$. The supernatant was discarded, and this step was repeated.

S7. The pellet was resuspended with MIB $(70 \mathrm{mM}$ sucrose, $210 \mathrm{mM}$ mannitol, $10 \mathrm{mM}$ HEPES, $1 \mathrm{mM}$ EDTA, 1 mM PMSF, pH 7.5) and shaken for 1 hour at $4{ }^{\circ} \mathrm{C}$ gently.

S8. The suspension was homogenized using a Teflon dounce homogenizer, and the homogenization time for the sufficient lysis of the cell had to be determined, as over-lysis results in burst mitochondria. Empirically, 20 strokes with the homogenizer were sufficient to burst the protoplast.

S9. The mixture was centrifuged at $600 \mathrm{~g}$ for $10 \mathrm{~min}$ at $4{ }^{\circ} \mathrm{C}$, and the supernatant was transferred into new EP tube and centrifuged at 11,000 g for $10 \mathrm{~min}$ at $4{ }^{\circ} \mathrm{C}$.

S10. The supernatant was discarded, and the mitochondria were resuspended in storage solution $(0.4$ $\mathrm{M}$ mannitol, $50 \mathrm{mM}$ Tris, $1 \mathrm{mM}$ EDTA, $5 \mathrm{mM} \mathrm{KCl}$, 5\% DMSO, $\mathrm{pH} 7.4$ ).

\section{Mitochondrial purity determination}

Different components of the cell were prepared, including total protoplast, nucleus, mitochondria and the cytoplasm, which were resuspended in lysis buffer $(100 \mathrm{mM}$ Tris-HCl, pH 8.0, $150 \mathrm{mM} \mathrm{NaCl}, 5$ mM EGTA, $5 \mathrm{mM}$ EDTA, $10 \mathrm{mM}$ DTT, 0.5\% [v/v] Triton X-100). The samples were mixed with loading buffer $(250 \mathrm{mM}$ Tris-HCL, $10 \%$ SDS, $0.5 \%$ BPB, $50 \%$ glycerol, $5 \%$ 2-ME) and were denatured at $98{ }^{\circ} \mathrm{C}$ for $10 \mathrm{~min}$. The proteins were separated by a $10 \%$ SDS-page gel. For the western blot, the proteins were transferred onto a PVDF membrane (BioRad, Hercules, CA, USA). Western blots were conducted by using the following primary antibodies and dilutions: anti-rubisco 1:5000, anti-VDAC 1:2000, anti-histone 1:5000, anti-COX II, 1:1000 and anti-catalase 1:1000. This was followed by incubation with the corresponding secondary antibody at a 1:5000 dilution. Signals were visualized by chemiluminescence (Bio-Rad).

For the genome purity assessment, the protoplasts and mitochondria were resuspended in CTAB buffer (2\% CTAB, 20 mM Na ${ }_{2}$ EDTA, $100 \mathrm{mM}$ Tris-HCl, $1.4 \mathrm{M}$ $\mathrm{NaCl}, \mathrm{pH} 8.0)$ at $60{ }^{\circ} \mathrm{C}$ for $1 \mathrm{~h}$. The DNA was extracted according to the standard protocol. The DNA concentration was measured by a Nanodrop 2000c and diluted to $15 \mathrm{ng} / \mathrm{ul}$. The PCR reaction was performed with $2 \times$ Taq plus master mix (Vazyme Biotech Co, P111/P112, Nanjing, China) according to the manufacturer's instructions. The forward primer actin-F (5'-AACTGAAACCCCCAT GTCCC-3') and reverse primer actin-R (5'-TGCAGA ACGGAAAAGTCCCA-3') were used for the amplification of the actin domain (actin, LOC_Os03g61970). The forward primer cox III-F (5'-GACAAATGGGAATAA CCGAA- $3^{\prime}$ ) and reverse primer cox III-R (5'-GGGGAA GGAAAAACGAGCAG-3') were used for the amplification of the cox III domain (cox III, LOC_Osm1g00110.1). The forward primer rubisco-F (5'-ATGTCACCACAA ACAGAAAC- $\left.3^{\prime}\right)$ and reverse primer rubisco- $\mathrm{R}\left(5^{\prime}-\mathrm{CTA}\right.$ GCTATCTAGTTTATCTA- $3^{\prime}$ ) were used for the amplification of the rubisco large subunit domain (rubisco, OrsajCp033). The PCR conditions were as follows: $95{ }^{\circ} \mathrm{C}$ for $5 \mathrm{~min}$, followed by 35 cycles of $95{ }^{\circ} \mathrm{C}$ for $15 \mathrm{~s}, 55^{\circ} \mathrm{C}$ for $15 \mathrm{~s}$, and $72{ }^{\circ} \mathrm{C}$ for $30 \mathrm{~s}$. The PCR products were separated in a $2 \%$ agarose gel and visualized by a gel imaging system (GE).

\section{Intact mitochondria examination}

The extracted mitochondria were incubated with proteinase $\mathrm{K}(0 \mu \mathrm{g} / \mathrm{ml}, 5 \mu \mathrm{g} / \mathrm{ml}, 20 \mu \mathrm{g} / \mathrm{ml}, 50 \mu \mathrm{g} / \mathrm{ml})$ or thermolysin $(0 \mu \mathrm{g} / \mathrm{ml}, 10 \mu \mathrm{g} / \mathrm{ml}, 50 \mu \mathrm{g} / \mathrm{ml}, 100 \mu \mathrm{g} / \mathrm{ml})$ at $4{ }^{\circ} \mathrm{C}$ for $20 \mathrm{~min}$. $\mathrm{CaCl}_{2}$ was added to the thermolysin to a final concentration of $0.5 \mu \mathrm{M}$. All of the reactions were quenched by adding EDTA to a final concentration of $5 \mathrm{mM}$, after which SDS-page electrophoresis was performed, and western blotting was conducted as described above.

\section{Transmission electron microscopy observation}

The sample preparation of mitochondria for transmission electron microscopy is described as follows: (i) the isolated mitochondria were fixed in $2 \%$ glutaraldehyde (2.5\% glutaraldehyde, $100 \mathrm{mM} \mathrm{NaH} \mathrm{PO}_{4} \mathrm{H}_{2} \mathrm{O}, 80 \mathrm{mM}$ $\left.\mathrm{Na}_{2} \mathrm{HPO}_{4} 12 \mathrm{H}_{2} \mathrm{O}\right)$ overnight and washed in phosphate buffer $\left(100 \mathrm{mM} \mathrm{NaH} \mathrm{PO}_{4} \mathrm{H}_{2} \mathrm{O}, 80 \mathrm{mM} \mathrm{Na}_{2} \mathrm{HPO}_{4} 12 \mathrm{H}_{2} \mathrm{O}\right)$ 5 times for $20 \mathrm{~min}$ each; (ii) the sample was then fixed with $1 \%$ osmic acid (1\% osmic acid, $100 \mathrm{mM}$ 
$\mathrm{NaH}_{2} \mathrm{PO}_{4} \mathrm{H}_{2} \mathrm{O}, 80 \mathrm{mM} \mathrm{Na} \mathrm{HPO}_{4} 12 \mathrm{H}_{2} \mathrm{O}$ ) for $2 \mathrm{~h}$, followed by 5 washes in phosphate buffer for 30 min each; (iii) the sample was dehydrated in ethanol at increasing concentrations of $15 \%, 30 \%, 50 \%, 70 \%, 80 \%, 85 \%, 90 \%$, and $95 \%$ successively, 20 min each; (iv) the sample was dehydrated in ethanol 2 times for 45 min each; (v) the sample was treated with a mix of ethanol and propylene oxide (the ratio was 1:1) for $30 \mathrm{~min}$; (vi) the sample was treated with propylene oxide and spur resin (the ratios were $3: 1,1: 1$, and 1:3, with each different ratio used for $12 \mathrm{~h}$ in that order); (vii) the sample was embedded in spur resin and incubated in an oven for $8 \mathrm{~h}$ at $40^{\circ} \mathrm{C}$, followed $60^{\circ} \mathrm{C}$ for $1-2$ days; (viii) the sample was sliced with an ultrathin microtome, followed by staining, washing, and drying; (ix) the nickel net that was loaded with the sample was observed with a JEM-1400 Plus Transmission Electron Microscope.

\section{Mitochondrial membrane potential analysis}

For the protoplasts, the suspension was centrifuged at $600 \mathrm{~g}$ for $3 \mathrm{~min}$, and the pellets were resuspended in W5 solution and diluted to an appropriate concentration (to ensure no cell stacking in the light microscopy). The protoplast was incubated with $1 \mathrm{X} \mathrm{JC}-1$ staining solution (6,6'-tetrachloro-1, 1', 3,3'-tetraethylbenzimidazole-carbocyanide iodine) (1:50) (Beyotime) for $20 \mathrm{~min}$, following 20 min CCCP (carbonyl cyanide m-chlorophenylhydrazone) treatment. The dual emission fluorescence was detected by fluorescence microscopy (FV1000 confocal system). Briefly, the JC-1 monomer was detected using a $514 \mathrm{~nm}$ excitation wavelength and a $529 \mathrm{~nm}$ emission wavelength. JC-1 aggregates were detected using a $525 \mathrm{~nm}$ excitation wavelength and a $590 \mathrm{~nm}$ emission wavelength. For the fluorescent enzyme labeling detection, three replicates were used for each sample, which were loaded into a black enzyme plate (Costar) for dual fluorescence detection by a mono-photometric system (Cytation 3 multimode reader, PengDe).

For the mitochondria, the pellets were resuspended in storage buffer and treated with CCCP for $5 \mathrm{~min}$, followed by incubation with $0.1 \mathrm{X} \mathrm{JC}-1$ staining solution for $5 \mathrm{~min}$. The dual fluorescence was detected by a mono-photometric system, similar to the protoplasts.

\section{Mitochondrial complex activity detection}

The activities of mitochondrial complex I, complex II, and complex IV were detected by a Nanodrop 2000c using the MRC (mitochondrial respiration chain) complex I, complex II and complex IV activity assay kit (Solarbio Life Sciences, Beijing, China). Briefly, the protoplasts were treated with rotenone $(0 \mu \mathrm{g} / \mathrm{ml}, 3 \mu \mathrm{g} /$ $\mathrm{ml}, 6 \mu \mathrm{g} / \mathrm{ml}, 9 \mu \mathrm{g} / \mathrm{ml})$, malonate $(0 \mathrm{mg} / \mathrm{ml}, 0.5 \mathrm{mg} / \mathrm{ml}$, $2.5 \mathrm{mg} / \mathrm{ml}, 5 \mathrm{mg} / \mathrm{ml})$ and sodium azide $(0 \%, 0.1 \%, 1 \%$,
$2 \%$ ) for $30 \mathrm{~min}$ at $25{ }^{\circ} \mathrm{C}$, respectively. Then, the mitochondria isolation procedure followed, as outlined above. All of the assays were performed according to the manufacturer's instructions.

\section{Supplementary information}

Supplementary information accompanies this paper at https://doi. org/10.1186/s13007-020-00690-6.

Additional file 1: Figure S1. Digestion pattern of thermolysin treated mitochondira. The isolated mitochondria were digested with an increasing thermolysin concentration. VDAC and cytochrome C oxidase II (COXII) were examined as the marker proteins of the mitochondrial outer membrane and inner membrane, respectively. Figure S2. Protoplasts isolated from calli. a The calli were cultured on the subculture medium. b The protoplasts were isolated from the rice callus, which showed the complete degradation of cell wall and the spherical cell shape. Bar, $10 \mu \mathrm{M}$. Figure S3. Over-lysis of the mitochondria. Over homogenization of the protoplast suspension can cause the mitochondria burst. Black arrows indicate the broken mitochondria membrane, white arrows indicate the inner membrane cristae of intact mitochondria. Bar, $500 \mathrm{~nm}$.

\section{Abbreviations}

ETC: Electron transfer chain; MIB: Mitochondrial isolation buffer; FLB: Full lysis buffer; VDAC: Voltage-dependent anion channels; COX II: Cytochrome C oxidase II; NAD3: NADH dehydrogenase subunit 3; CCCP: Carbonyl cyanide 3-chlorophenylhydrazone.

\section{Acknowledgements}

We thank Yunping Chen for constructive suggestions, Ting Liang for the protoplast isolation and Wenxuan Zou for the transmission electron microscope assistance.

\section{Authors' contributions}

$J H$ and $Y X$ conceived and designed the experiment, YHX, XYL, JH, LP, DL, QZ performed the experiments, YHX wrote the manuscript, $H X, Z D, F Y$ and $J H$ revised the manuscript. All authors read and approved the manuscript.

\section{Funding}

This work was supported by funds from National Natural Science Foundation of China (31371698, 31670310, and 31871592), the Foundation for Innovative Research Groups of Hubei Province (ZRQT2020000031) and Suzhou science and technology project (SNG2017061), and the State Key Laboratory for Conservation and Utilization of Subtropical Agro-Bioresources (No. SKLCUSAb201717), and the Key Laboratory of Ministry of Education for Genetics, Breeding and Multiple Utilization of Crops, College of Crop Science, Fujian Agriculture and Forestry University (PTJH12004).

\section{Availability of data and materials}

All the data generated or analyzed during this study are included within this article.

Ethics approval and consent to participate

Not applicable.

\section{Consent for publication \\ Not applicable.}

\section{Competing interests}

The authors declare that they have no competing interests.

\section{Author details}

${ }^{1}$ State Key Laboratory of Hybrid Rice, Engineering Research Center for Plant Biotechnology and Germplasm Utilization of Ministry of Education, College of Life Sciences, Wuhan University, Wuhan 430072, Hubei, China. ${ }^{2}$ College of Life Sciences, Sichuan University, Chengdu 610065, Sichuan, China. 
Received: 27 August 2020 Accepted: 27 October 2020

Published online: 04 November 2020

\section{References}

1. Lane N, Martin W. The energetics of genome complexity. Nature. 2010;467(7318):929-34.

2. Lodish H BA, Zipursky SL, et al. Molecular Cell Biology. 4th edition. New York: W H Freeman. Organelles of the Eukaryotic Cell; 2000.

3. Bullerwell CE, Gray MW. Evolution of the mitochondrial genome: protist connections to animals, fungi and plants. Curr Opin Microbiol. 2004;7(5):528-34.

4. Gualberto JM, Mileshina D, Wallet C, Niazi AK, Weber-Lotfi F, Dietrich A. The plant mitochondrial genome: dynamics and maintenance. Biochimie. 2014;100:107-20.

5. Anderson S, De Bruijn MH, Coulson AR, Eperon IC, Sanger F, Young IG. Complete sequence of bovine mitochondrial DNA conserved features of the mammalian mitochondrial genome. J Mol Biol. 1982;156:683-717.

6. Castora FJ. Mitochondrial function and abnormalities implicated in the pathogenesis of ASD. Prog Neuropsychopharmacol Biol Psychiatry. 2019;92:83-108.

7. Peng XKW, Hu C, Zhu Y, Wang T, Yang J, Tong J, Li S, Zhu Y. The mitochondrial gene orfH79 plays a critical role in impairing both male gametophyte development and root growth in CMS-Honglian rice. BMC Plant Biol. 2010;10:125.

8. Liberatore KL, Dukowic-Schulze S, Miller ME, Chen C, Kianian SF. The role of mitochondria in plant development and stress tolerance. Free Radical Biol Med. 2016;100:238.

9. Levings CS. the texas cytoplasm of maize-cytoplasmic male sterility and disease susceeptibility. Science. 1990;250:942-7.

10. Wang X, Su B, Lee HG, Li X, Perry G, Smith MA, et al. Impaired balance of mitochondrial fission and fusion in Alzheimer's disease. J Neurosci. 2009;29(28):9090-103

11. Exner N, Treske B, Paquet D, Holmstrom K, Schiesling C, Gispert S, et al. Loss-of-function of human PINK1 results in mitochondrial pathology and can be rescued by parkin. J Neurosci. 2007;27(45):12413-8.

12. Nunnari J, Suomalainen A. Mitochondria: in sickness and in health. Cell. 2012;148(6):1145-59.

13. Gammage PA, Viscomi C, Simard ML, Costa ASH, Gaude E, Powell CA, et al. Genome editing in mitochondria corrects a pathogenic mtDNA mutation in vivo. Nat Med. 2018:24(11):1691-5.

14. Van Vuuren AJ, Appeldoorn E, Odijk H, Yasui A, Jaspers NG, Bootsma D, Hoeijmakers $\mathrm{JH}$. Evidence for a repair enzyme complex involving ERCC1 and complementing activities of ERCC4, ERCC11 and xeroderma pigmentosum group F. EMBO J. 1993;12(9):3693-701.

15. Luo D, Xu H, Liu Z, Guo J, Li H, Chen L, et al. A detrimental mitochondrialnuclear interaction causes cytoplasmic male sterility in rice. Nat Genet. 2013;45(5):573-7.

16. Abad AR, Mehrtens BJ, Mackenzie SA. Specific expression in reproductive tissues and fate of a mitochondrial sterility-associated portein incytoplasmic male-sterile bean. Plant Cell. 1995;7:271-85.

17. Pring DR, Chen W, Tang HV, Howad W, Kempken F. Interaction of mitochondrial RNA editing and nucleolytic processing in the restoration of male fertility in sorghum. Curr Genet. 1998;33:429-36.

18. Hu J, Wang K, Huang W, Liu G, Gao Y, Wang J, et al. The rice pentatricopeptide repeat protein RF5 restores fertility in Hong-Lian cytoplasmic male-sterile lines via a complex with the glycine-rich protein GRP162. Plant Cell. 2012;24(1):109-22.
19. Liu F, Cui X, Horner HT, Weiner H, Schnable PS. Mitochondrial aldehyde dehydrogenase activity is required for male fertility in maize. Plant Cell. 2001;13(5):1063-78.

20. Tang H, Luo D, Zhou D, Zhang Q, Tian D, Zheng X, et al. The rice restorer Rf4 for wild-abortive cytoplasmic male sterility encodes a mitochondriallocalized PPR protein that functions in reduction of WA352 transcripts. Mol Plant. 2014;7(9):1497-500.

21. Ding B, Hao M, Mei D, Zaman QU, Sang S, Wang H, et al. Transcriptome and hormone comparison of three cytoplasmic male sterile systems in brassica NAPUS. Int J Mol Sci. 2018;19(12):1-12.

22. Dan Z, Chen Y, Xu Y, Huang J, Huang J, Hu J, et al. A metabolome-based core hybridisation strategy for the prediction of rice grain weight across environments. Plant Biotechnol J. 2018; pp. 1-8.

23. Eubel $\mathrm{H}$, Heazlewood JL, Millar AH. Isolation and subfractionation of plant mitochondria for proteomic analysis. Methods Mol Biol (Clifton, NJ). 2007:355:49-62.

24. Aviram R, Manella G, Kopelman N, Neufeld-Cohen A, Zwighaft Z, Elimelech $\mathrm{M}$, et al. Lipidomics analyses reveal temporal and spatial lipid organization and uncover daily oscillations in intracellular organelles. Mol Cell. 2016:62(4):636-48.

25. Shen J, Fu J, Ma J, Wang X, Gao C, Zhuang C, et al. Isolation, culture, and transient transformation of plant protoplasts. Curr Protoc Cell Biol. 2014;63:2-8.

26. Yoo SD, Cho YH, Sheen J. Arabidopsis mesophyll protoplasts: a versatile cell system for transient gene expression analysis. Nat Protoc. 2007;2(7):1565-72.

27. Froehlich J. Studying Arabidopsis envelope protein localization and topology using thermolysin and trypsin proteases. Methods Mol Biol. 2011;774:351-67.

28. Wojtyla L, Kosmala A, Garnczarska M. Lupine embryo axes under salinity stress. II. Mitochondrial proteome response. Acta Physiol Plant. 2013;35(8):2383-92

29. Chazotte B. Labeling mitochondria with JC-1. Cold Spring Harb Protoc. 2011;2011(9):1103-1104.

30. Hu Hao JT, Fang Yijun, Fu Yajuan. Mitochondrial damage and mtDNA release induced by sodium azide. China academic journal. 2019;6.

31. Day DA, Whelan J, Millar AH, Siedow JN, Wiskich JT. Regulation of the alternative oxidase in plants and fungi. J Plant Physiol. 1995;22:497-509.

32. Jenny A WaRJ. Agonist-induced calcium and oxidative stress responses in endothelial cells. Biochemical Society Transactions. 2003; Vol 31, part 5.

33. Zaghmout OF, Trolinder NL. Factors affecting transient gene expression in protoplasts isolated from veery slowly growing embryogenic callus cultures of wheat (triticum aestivum L). Theor Appl Genet. 1993;86:721-30.

34. McCormack JC, Simon AE. Callus Cultures of Arabidopsis. Curr Protocols Microbiol. 2005;16D.1.1-16D.1.9.

35. Liqing Z, Bochu W, Jing Z, Lingxi C, Chuanyun D, Chuanren D. Protoplast isolation of callus in Echinacea augustifolia. Colloids Surf B Biointerfaces. 2005;44(1):1-5.

\section{Publisher's Note}

Springer Nature remains neutral with regard to jurisdictional claims in published maps and institutional affiliations. 